\title{
Méthode de dosage de l'aflatoxine $M$ dans le lait et les produits laitiers
}

\author{
par \\ P. LAFONT, M. SIRIWARDANA, J. JACQUET \\ M. GAILLARDIN et J. SARFATI*
}

\section{INTRODUCTION}

La présence d'aflatoxine $\mathbf{M}$ dans le lait et les produits laitiers pose des problèmes de santé publique à propos desquels l'inquiétude des hygiénistes ne cesse de croître. Aussi, des normes précisant des teneurs maximales permissibles ont-elles été données en certains pays, ou sont en préparation dans d'autres. Pour les nations dont les services sanitaires ont adopté zéro comme tolérance, il est évident qu'ils s'en remettent, en fait, à la limite de sensibilité des analyses qui sont effectuées.

De toutes façons, dans tous les cas, toute action de prévention et de contrôle repose sur l'existence d'une bonne méthode de recherche et de dosage de ces mycotoxines.

Or, le dosage des difuranocoumarines $\mathbf{M}$ dans le lait et ses dérivés se montre plus délicat que celui des formes B et $G$ dans d'autres denrées. On a besoin d'une finesse plus grande, car il faut apprécier de façon sûre des concentrations beaucoup plus faibles ; les extraits de produits laitiers contiennent fréquemment des substances qui viennent former des artefacts sur les chromatogrammes, si on n'a pas réussi à s'en débarrasser auparavant. Il y a intérêt, enfin, à ce que la méthode soit polyvalente, et puisse s'appliquer aussi bien à tous les produits laitiers, même ceux qui sont acides, qu'au lait lui-même.

Après de nombreux essais, qui ont porté sur la mise au point de chacun des temps de l'analyse, nous proposons la méthode ci-dessous.

* I.N.S.E.R.M., Service de Microbiologie Appliquée à l'Alimentation et à la Nutrition, 44, chemin de Ronde - 78110 Le Vésinet. 


\section{MATERIELS ET PRODUITS NECESSAIRES}

\section{A. Matériel}

Ampoules à décanter de $500 \mathrm{ml}$.

Colonne à chromatographie à robinet (diamètre intérieur $18 \mathrm{~m} / \mathrm{m}$ ).

Evaporateur rotatif avec condensateur à neige carbonique, ballon d'évaporation de $1000 \mathrm{ml}$ et fiole conique de $250 \mathrm{ml}$.

Seringues Hamilton ou Terumo ou encore micropipettes microcaps (Drummond Scientific $\mathrm{C}^{\circ}$ ).

Plaques de chromatographie Merck Si 60 (5721).

Papiers filtre Whatmann $\mathrm{n}^{\circ} 1$.

Lampe Philipps HPW 125.

Photodensitomètre PHI 5, Sté $\mathrm{A}^{\text {me }}$ Vernon, Paris.

\section{B. Produits et réactifs}

Alumine basique, Aluminoxid 90, aktiv-basisch Merck 70-230 mesh.

Eau distillée en appareil de verre.

Celite 545.

Sulfate de sodium RPE anhydre.

Isopropanol, méthanol, chloroforme, toluène, éther éthylique, acétone, (qualité Merck pour analyses, Carlo-Erba RPE, ou similaire).

Epichlorhydrine du glycérol pure pour analyses.

Aflatoxines $M$ de référence cristallisées Serva-Feinbiochem Heildelberg.

\section{TECHNIQUE ANALYTIQUE}

\section{A. Préparations initiales}

$1^{\circ}$ Colonne a chromatographie (à préparer soi-même et avec le plus grand soin, aucune colonne disponible actuellement dans le commerce ne donnant, à notre connaissance, de résultats satisfaisants). On introduit successivement dans la colonne, $10 \mathrm{~g}$ de sulfate de sodium anhydre pulvérulent, un disque de papier filtre, $1 \mathrm{~g}$ d'alumine basique. On remplit partiellement la colonne avec du toluène ; par une légère agitation avec une tige de verre on élimine l'air contenu dans l'alumine. On superpose, alors, à la couche d'alumine un disque de papier-filtre, puis $2 \mathrm{~g}$ de silice en suspension dans le toluène. Le matériel introduit dans la colonne est tassé en exerçant une pression avec de l'azote. Un disque de papier-filtre est déposé sur la silice, puis recouvert de $2 \mathrm{~g}$ de sulfate de sodium anhydre. 
20 LIQUide D'ÉLUTION DE LA CHROMATOGRAPHIE EN COUCHE MINCE (doit être préparé $2 h$ avant son emploi). Mélanger en volumes les quantités suivantes :

- éther éthylique 90

- méthanol 3

- épichlorhydrine 6

- eau

3o PréParation des échantillons pour l'analyse.

$50 \mathrm{ml}$ de lait sont portés au bain-marie bouillant pendant $5 \mathrm{~min}$, puis refroidis à la température du laboratoire.

Les laits fermentés, comme le yaourt, seront préparés comme les laits liquides.

S'il s'agit de poudre de lait, on pèse $10 \mathrm{~g}$ que l'on mettra en solution dans $30 \mathrm{ml}$ d'eau distillée.

Pour les fromages, peser $20 \mathrm{~g}$ que l'on broiera dans $10 \mathrm{ml}$ d'eau dans un flacon de $250 \mathrm{ml}$.

\section{B. Description de la méthode}

1. Extraction des aflatoxines. Introduire l'échantillon tel qu'il a été préparé au $\S$ II A $3^{\circ}$ dans une ampoule à décanter de $500 \mathrm{ml}^{*}$. Y verser ensuite lentement et en agitant $60 \mathrm{ml}$ d'isopropanol par apports successifs de quelques millilitres. Le mélange est, alors, secoué vigoureusement pendant $1 \mathrm{~min}$. On ajoute, ensuite, $50 \mathrm{ml}$ de méthanol, $10 \mathrm{~g}$ de célite, $250 \mathrm{ml}$ de chloroforme, le mélange étant soumis à une agitation violente de $1 \mathrm{~min}$ après l'introduction de chacun de ces produits. Après un repos de 5 à $10 \mathrm{~min}$, la phase liquide inférieure, limpide, est soutirée, filtrée sur papier supportant $10 \mathrm{~g}$ de sulfate de sodium anhydre. On prélève $300 \mathrm{ml}$ du filtrat. On les concentre dans un appareil rotatif d'évaporation sous vide, muni d'un condenseur à neige carbonique, la température du bain-marie étant réglée à $45^{\circ} \mathrm{C}$. Lorsqu'il reste environ $5 \mathrm{ml}$ de liquide (il s'agit alors d'un mélange riche en isopropanol), on introduit dans le ballon d'évaporation $30 \mathrm{ml}$ de chloroforme et on poursuit la concentration jusqu'à environ $0,5 \mathrm{ml}$. Ce résidu est additionné de $30 \mathrm{ml}$ de toluène. On concentre à nouveau à $0,5 \mathrm{ml}$; on introduit encore une fois $30 \mathrm{ml}$ de toluène dans le ballon d'évaporation et on concentre à $5 \mathrm{ml}$.

2o PuRification de L'extrait Par chromatographie sur COLONNE. L'extrait obtenu au $\S$ précédent II B $1^{\circ}$ est versé dans la colonne à chromatographie préparée selon le mode précisé au $\S$ II A $1^{\circ}$; on lave le ballon d'extraction avec $15 \mathrm{ml}$ de toluène que l'on transporte dans la même colonne. On procède ensuite à des élutions successives par $50 \mathrm{ml}$ de toluène, par $50 \mathrm{ml}$ de chloroforme et par $100 \mathrm{ml}$ du mélange chloroforme-acétone $(4 / 1, \mathrm{v} / \mathrm{v})$.

* Dans le cas du fromage, c'est dans le flacon qui a servi au broyage que se font les adjonctions d'isopropanol, méthanol, célite et chloroforme, conformément aux indications du § II B 1. Après repos, on récupère la phase supérieure non aqueuse qui est filtrée (voir $10^{\mathrm{e}}$ ligne du paragraphe) ; le reste des manipulations sans changement. 
L'éluat obtenu avec cette dernière phase est concentré à moins de $1 \mathrm{ml}$ dans un appareil rotatif d'évaporation fonctionnant dans les mêmes conditions que celles décrites précédemment $\left(\mathrm{B} 1^{\circ}\right)$. Le résidu et le produit de lavage de la fiole d'évaporation par $1 \mathrm{ml}$ de chloroforme sont transportés dans un tube à hémolyse. Le solvant est évaporé sous courant d'azote (le tube étant placé dans un bain-marie à $45^{\circ} \mathrm{C}$ ) jusqu'à $100 \mu \mathrm{l}$.

$3^{\circ}$ Dosage des aflatoxines EN CHROMATOGRAPHIE SUR COUCHE MINCE. On dépose sur gel de silice activé $\left(110^{\circ} \mathrm{C} / 20 \mathrm{~min}\right)$ :

- $20 \mu \mathrm{l}$ de l'extrait ;

- des volumes égaux de solutions d'aflatoxine M1 contenant $0,1-0,2-0,3-0,4=0,5-0,6$ et $0,7 \mathrm{ng}$ de la toxine ;

- en un même point, $10 \mu \mathrm{l}$ de l'extrait et $0,5 \mathrm{ng}$ d'aflatoxine M1 (témoin interne). On a donc disposé 9 points de dépôt.

Le développement est réalisé sur $12 \mathrm{~cm}$ environ, en cuve à atmosphère non saturée, en utilisant la phase éluante préparée selon le § II A $2^{\circ}$. Le chromatogramme est examiné sous irradiation ultra-violette.

La fluorescence de la difuranocoumarine $M$ est bleue violacée. Son Rf est $2,4 \pm 0,2$.

Si l'intensité de la fluorescence de l'aflatoxine M1 contenue dans les $20 \mu$ l déposés d'extrait excède celle du témoin le plus concentré $(0,7 \mathrm{ng})$, on recommence l'opération en faisant des dilutions de l'extrait. Si aucune fluorescence n'est décelable au niveau de l'extrait, on procède à un nouvel essai. Pour les faibles concentrations d'aflatoxine M1 dans l'échantillon, le dosage ne peut être réalisé que par comparaison visuelle de l'intensité de fluorescence des spots. Pour de plus fortes concentrations, supérieures à $100 \mu \mathrm{g} / \mathrm{k}$, on peut utiliser un photodensitomètre*.

\section{4o Méthode de confirmation}

La présence d'aflatoxine M1 est confirmée, sur les chromatographies en couche mince, par la méthode de Przybylski [4] modifiée selon van Egmond et al. [7] : traitement de chacune des taches fluorescentes d'aflatoxine témoin et des taches de Rf analogues par $5 \mu$ l du mélange hexane-acide trifluoroacétique $(4 / 1 ; \mathrm{v} / \mathrm{v}$ ) (chauffage $5 \mathrm{~min}$ à $75^{\circ} \mathrm{C}$ après avoir recouvert avec une plaque de verre) nouveau développement chromatographique.

\section{Calcul des résultats}

Il faut se rappeler que toute l'aflatoxine se trouve pratiquement, après décantation (cf. $\S \mathrm{B} 1^{\circ}$ ) dans les solvants, l'eau n'en retenant que des traces infimes. Du fait que dans les temps ultérieurs de

* Il est nécessaire de s'assurer, grâce à des manipulations témoins, de l'absence de contamination accidentelle du matériel utilisé (verrerie, micro-seringues). 
l'analyse, on n'emploie, pour des raisons de facilité de soutirage, qu'une partie aliquote, il y a lieu de rapporter les valeurs obtenues en chromatographie sur couche mince à l'ensemble de ce qui était dissous dans la phase solvants organiques.

La quantité présente dans les $50 \mathrm{ml}$ de lait de consommation ou de lait fermenté de départ sera donc exprimée par :

$$
\text { quantité estimée sur le chromatogramme } \times \frac{345}{300}(=1,15)
$$

Pour les laits en poudre, pour les $10 \mathrm{~g}$ de départ, il s'agira de :

$$
\text { quantité lue sur chromatogramme } \times \frac{360}{300}(=1,2)
$$

Le même coefficient de 1,2 est appliqué pour les $20 \mathrm{~g}$ de fromage mis en œuvre.

\section{DISCUSSION}

L'originalité de la méthode repose sur plusieurs points. Il y a, d'abord, la réalisation d'une extraction de l'aflatoxine par trois solvants différents absolument nécessaire lorsque l'on a affaire à de très petites quantités, ce qui est le cas avec la difuranocoumarine $\mathbf{M}$, quand elle est présente dans le lait et les produits laitiers. D'une façon générale, l'analyste doit toujours avoir présente à l'esprit l'idée que, dans ce type de recherches, on ne dose jamais que ce que l'on a réussi à sortir du produit initial. On ne saurait donc apporter trop d'attention à ce premier temps du travail.

Il y a, ensuite, l'emploi de deux adsorbants dans la colonne de purification. La bonne préparation de celle-ci comme nous l'avons déjà signalé, est essentielle pour la réussite de l'ensemble.

Il y a, enfin, l'utilisation d'un nouveau liquide d'élution donnant d'excellents résultats dans la chromatographie sur couche mince.

Une des clefs du succès du dosage des aflatoxines $\mathrm{M}$ dans le lait nous paraît être le choix, lors de l'extraction, d'une technique et de solvants qui ne dégradent pas et ne précipitent pas les protéines.

Le grand avantage de la méthode que nous proposons est, tout d'abord, sa polyvalence : d'une part, elle est applicable à la recherche et au dosage d'aflatoxines autres que M1 et M2 ; d'autre part, elle permet une analyse, non seulement, du lait, mais aussi, de différents produits laitiers, dont les fromages et les laits fermentés.

La sensibilité de la méthode est grande : on reconnaît la présence de quelques nanogrammes d'aflatoxine M1 dans $1 \mathrm{~kg}$ de lait liquide, qu'il s'agisse de lait en nature ou de lait reconstitué à partir de poudre, (2,3 à 4,6 ng/ $\mathrm{kg}$ suivant les échantillons) et on apprécie de façon sûre des concentrations de la mycotoxine inférieures à $10 \mathrm{ng} / \mathrm{kg}$. 
Fiabilité de la méthode

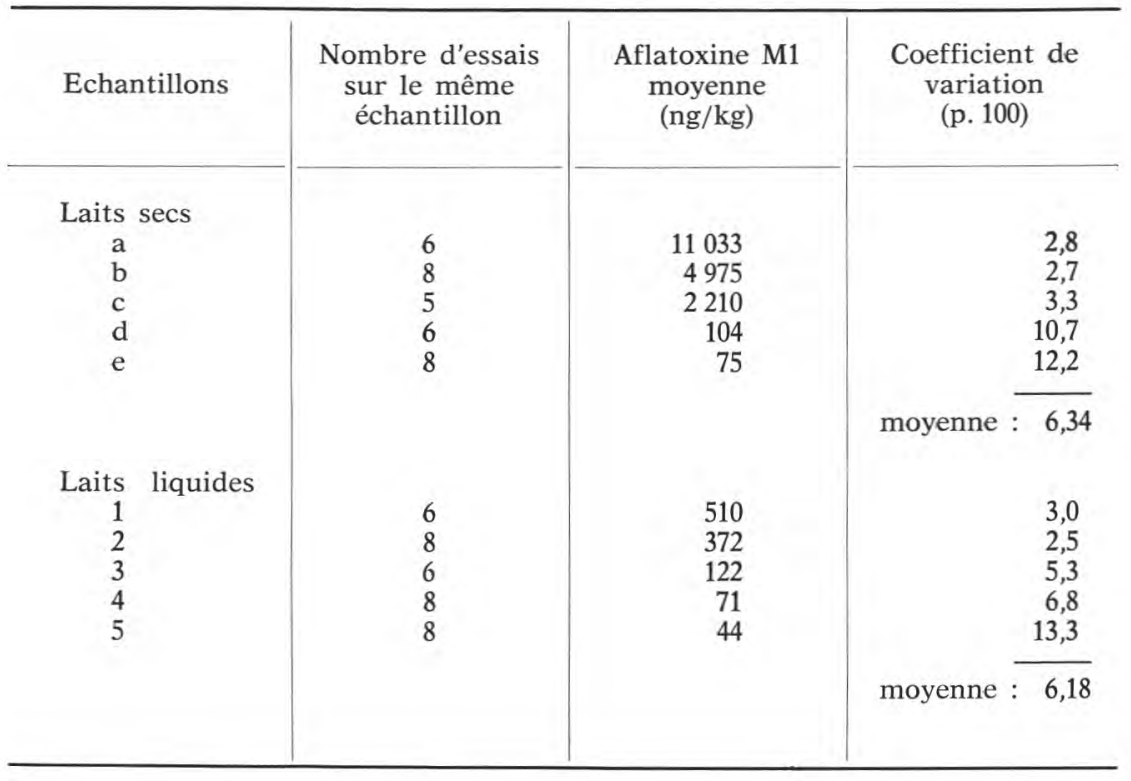

La fiabilité des dosages ressort du tableau ci-dessus, et s'avère excellente, surtout pour des analyses de ce genre. Dans des séries d'essais comparatifs, portant sur des laits naturellement contaminés, la méthode ici décrite a fourni des résultats meilleurs, soit en matière de sensibilité, soit en matière de fiabilité, que ceux obtenus en appliquant d'autres méthodes, dont celle de Pons et al. modifiée [3, 2], celle de Gauch et al. [1], celle de Tuinstra et Brongeest [6], celle de Stubblefield [5].

\section{Rés u mé}

La méthode de dosage des aflatoxines $\mathrm{M}$ que nous décrivons est originale par l'emploi d'une colonne de chromatographie de purification contenant deux adsorbants, la réalisation d'une triple extraction des flavacoumarines, l'utilisation d'un nouveau liquide d'élution en chromatographie sur couche mince de silice. Elle se recommande par sa polyvalence (elle est applicable au dosage des différentes aflatoxines), ses possibilités d'utilisation dans l'analyse non seulement du lait mais aussi d'autres produits laitiers, sa fidélité (coefficient moyen de variation de 6,26 p. 100), sa sensibilité (moins de $10 \mathrm{ng} / \mathrm{kg}$ de lait en nature ou reconstitué à partir de poudre).

Mots clés : Lait - Fromages - Aflatoxines - Aflatoxines M1. 


\section{S u $\mathrm{m} \mathrm{m}$ a r y}

\section{AFLATOXINS M ASSAY IN MILK AND DAIRY PRODUCTS}

The assay method of aflatoxins $M$ here described is real new one by using two adsorbents in the cleaning chromatographic column, by using a triple extraction of the mycotoxins and by using also a new eluent for the thin layer chromatography. It is particularly interesting by its multiple applications (assays of several aflatoxins, use for the milk and also for other dairy products), by a high grade of reliability (mean standard error: 6,26 p.100) and by its sensibility (less than $10 \mathrm{ng} / \mathrm{kg}$ of liquid milk).

Key words: Milk - Dairy products - Aflatoxins - Aflatoxins M1.

\section{Bibliographie}

[1] Gauch (R.), Lenenberger (U.) and Baumgartner (E.) (1979). - Rapid and simple determination of aflatoxin M1 in milk in the low parts per $10^{12}$ range. J. of Chromatog., 178, 543-549.

[2] Lafont (P.), Lafont (J.), Mousset (S.) et Frayssinet (C.) (1980). - Etude de la contamination du lait de vache lors de l'ingestion de faibles quantités d'aflatoxine. Ann. Nutr. Alim., 34, 699-708.

[3] Pons (W.), Cucullu (A.) and Lee (L.) (1973). - Method for the determination of aflatoxin M1 in fluid milk and milk products. J.A.O.A.C., 56, 1431-1436.

[4] PRZybYLSKi (W.) (1975). - Formation of aflatoxin derivatives on thin layer chromatographic plates. J.A.O.A.C., 58, 163-164.

[5] Stubblefield (R.) (1979). - The rapid determination of aflatoxin M1 in dairy products. J. Am. Oil Chem. Soc., 56, 800-802.

[6] Tuinstra (L.) and Bronsgeest (J.) (1975). - Determination of aflatoxin M1 in milk at the parts per trillion level. J. of Chromatog., 111, 448-451.

[7] Van Egmond (H.), Paulsch (W.) and Shuller (P.) (1978). - Confirmatory test for aflatoxin $\mathrm{M} 1$ in a thin layer plate. J.A.O.A.C., 61, 809-812. 